\title{
Particle Assembly with Synchronized Acoustic Tweezers
}

\author{
Zhixiong Gong® and Michael Baudoin $\oplus^{*}$ \\ Université de Lille, CNRS, Centrale Lille, ISEN, Université Polytechniques Hauts-de-France, UMR 8520-IEMN, \\ International Laboratory LIA/LICS, F-59000 Lille, France
}

(Received 8 April 2019; revised manuscript received 12 July 2019; published 21 August 2019)

\begin{abstract}
The contactless selective manipulation of individual objects at the microscale is powerfully enabled by acoustical tweezers based on acoustical vortices [M. Baudoin et al., Sci. Adv. 5, eaav1967 (2019)]. Nevertheless, the ability to assemble multiple objects with these tweezers has not been demonstrated yet and is critical for many applications, such as tissue engineering or microrobotics. To achieve this goal, it is necessary to overcome a major difficulty: the high-intensity ring which ensures the trapping of inner particles at the core of the vortex beam is repulsive for particles located outside the trap. This prevents the assembly of multiple objects. In this paper, we show (in the Rayleigh limit and in two dimensions) that this problem can be overcome by trapping the target objects at the core of two synchronized vortices. Indeed, in this case, the destructive interference between neighboring vortices enables us to create an attractive path between the captured objects. The present work may pioneer the precise assembly and patterning of particles with multitweezers.
\end{abstract}

DOI: 10.1103/PhysRevApplied.12.024045

\section{INTRODUCTION}

Acoustical vortices $[1,2]$ have been attracting more and more attention for the selective manipulation of particles [3-9]. Unlike the ordinary zeroth-order Bessel beam [1012], acoustical vortices are helical waves spinning around a phase dislocation in the azimuthal direction, wherein the amplitude cancels. This minimum of the beam intensity surrounded by a bright ring of high intensity can serve as an acoustical trap for particles more dense and more stiff than the surrounding fluid and for sufficiently large cone angles [13]. Hence, the so-called cylindrical vortices (a separate variable solution of the Helmholtz equation in cylindrical coordinates [2]) are well suited for twodimensional (2D) particle trapping ( $x-y$ directions) $[4,7$, $13,14]$. Nevertheless, since the intensity of these beams is invariant along the $z$ axis, particles can only be pushed or pulled (in the so-called tractor beam configuration) [1519], but not stably trapped in this direction. Instead, it was first proposed theoretically [3] and then demonstrated experimentally [20] by Baresh et al. that the use of spherical vortices enables us to create three-dimensional (3D) traps with a one-sided transducer. These wavefields can be obtained by adding an axial focalization to cylindrical vortices [3]. Trapping of particles with such focalized vortices in air was also demonstrated by Marzo et al. [5]. Alternatively, Riaud et al. [21] proposed to tame the degeneracy of a vortex beam between an anisotropic and an isotropic medium to create a $3 \mathrm{D}$ trap. More recently, it was shown

\footnotetext{
*michael.baudoin@univ-lille.fr
}

both theoretically and experimentally [22-29], that acoustical vortices can also be used to control the rotation of particles by using the pseudo-angular-momentum carried by these helical structures. Especially, the reversals of radiation torque in three dimensions have been studied on the physical mechanism and parameter conditions [26]. It is also noteworthy to underline that the calculations of the force and torque exerted by a vortex beam, initially calculated for spherical particles, have been recently extended to nonspherical particles with the use of the T-matrix method [18,26,30,31].

Experimentally, the approaches for the synthesis of acoustical vortices can be divided into three categories. The first one, that we refer to as the "active array method," relies on arrays of transducers whose phase and/or amplitude can be tuned to synthesize a vortex in the surrounding fluid $[1,4,7,20,21,28,32-36]$. The advantage of this method is that the vortex core (and thus the acoustical trap) can be moved electronically $[4,5,34,36]$ and multiple traps can be synthesized simultaneously [8]. The disadvantage is that this technique relies on a complex array of transducers and a programmable electronics, which become expensive and complex to miniaturize as the frequency is increased to trap smaller particles (especially in liquids wherein the sound speed is 5 times faster than in air). The second one, the "passive vortex-beam method," relies on passive devices, including spiral diffraction gratings [37-39], metasurfaces, or metastructure [40-42], which convert a plane or focused acoustic field into vortex beams. Finally, a third promising method, the "active hologram method," relies on the patterning of holographic electrodes at the surface of an 
active piezoelectric material. In this system, the hologram of the vortex is directly engraved in the shape of the electrodes, which can be patterned with classic photolithography techniques. This last method enables us to produce cheap, miniaturized, flat, transparent tweezers, which can be easily integrated in a classical microscopy environment to monitor displacement of small particles $[7,9,43]$.

It is important to note that the quest for the development of one-sided selective tweezers must be distinguished from the extensive work on acoustical traps based on standing waves [44-46]. Indeed, tweezers based on standing waves are versatile tools for sorting particles or collectively manipulating multiple objects, but the multiple nodes and antinodes prevent any selectivity. Indeed, since the wavefield is not localized in space (contrary to vortex beams wherein the energy is focalized), it is not possible to move one particle independently of other neighboring particles. In addition, standing waves can only be obtained by placing some transducers (or reflectors) all around the area of interest, which prevents the design of one-sided 3D tweezers.

While acoustical tweezers based on acoustical vortices have undergone tremendous progress toward miniaturization and improved selectivity on one side [9] and the simultaneous manipulation of multiple objects on the other side [8], one key operation is still missing for many applications, such as tissue engineering or microrobotics: the ability to assemble multiple objects. At first glance, this operation seems incompatible with the vortex-beam structure. Indeed, the particles are trapped at the center of a high-intensity ring, which ensures the trapping of inner particles, but is repulsive for particles located outside the trap. Thus, two particles located respectively inside and outside of the trap cannot be assembled. In this paper, we demonstrate theoretically that this problem can be overcome by trapping the objects to be assembled at the center of two synchronized vortex beams. Indeed, the destructive interference between these beams (when they are brought close to each other) creates an attractive path between the trapped particles. This demonstration is conducted in the limit of cylindrical vortices (transverse assembly), for cone angles equal to $90^{\circ}$ and in the long-wavelength approximation (i.e., for particles much smaller than the wavelength). With this calculation, we are also able to estimate the critical velocities for particle assembly depending on the properties of the trapped particles and surrounding fluid as well as the beam intensity.

\section{INTERACTION BETWEEN CYLINDRICAL VORTICES: INTENSITY AND PHASE}

In this section, we consider the interference in a transverse plane of fixed altitude $(z=0)$ between two cylindrical Bessel beams whose respective centers are located in $O_{1}(-s, 0)$ and $O_{2}(s, 0)$. Hence, the distance between the original cores of these two vortices is $2 s$ along the $x$ axis as shown in Fig. 1. In this configuration, the pressure field $p_{j}$ produced by the $j$ th vortex $(j=1$, left vortex; $j=2$, right vortex) is given by the equation

$$
p_{j}=A_{j} J_{m}\left(k_{\perp} r_{j}\right) e^{i\left(m_{j} \theta_{j}+k_{\|} z\right)} e^{i \beta_{j}},
$$

with $r_{j}$ the radial distance with respect to the origin of the $j$ th vortex beam; $A_{j}$ the beam amplitude; $m_{j}$ its topological charge; $\theta_{j}$ and $\beta_{j}$ the azimuthal and original phase angles, respectively; and finally, $k_{\perp}=k \sin (\gamma)$ and $k_{\|}=k \cos (\gamma)$ the transverse and axial wave numbers, with $k=\omega / c$ the wave number, $c$ the sound speed in the fluid, $\omega$ the angular frequency, and $\gamma$ the so-called cone angle. We can note that a cylindrical Bessel beam is entirely defined by its topological charge $m$ and the cone angle $\gamma$. For the sake of simplicity, we consider vortices of topological order $m=1$ (the order mostly considered to design acoustical tweezers since it provides the stiffest trap) and $\gamma=90^{\circ}$ (leading to $k_{\perp}=k$ and $k_{\|}=0$ ). Present results can be easily extended to any value of $m$ and $\gamma$ by following the same procedure as the one expressed below. Nevertheless, it is interesting to note that for cone angles $\gamma<90^{\circ}$, the axial component of

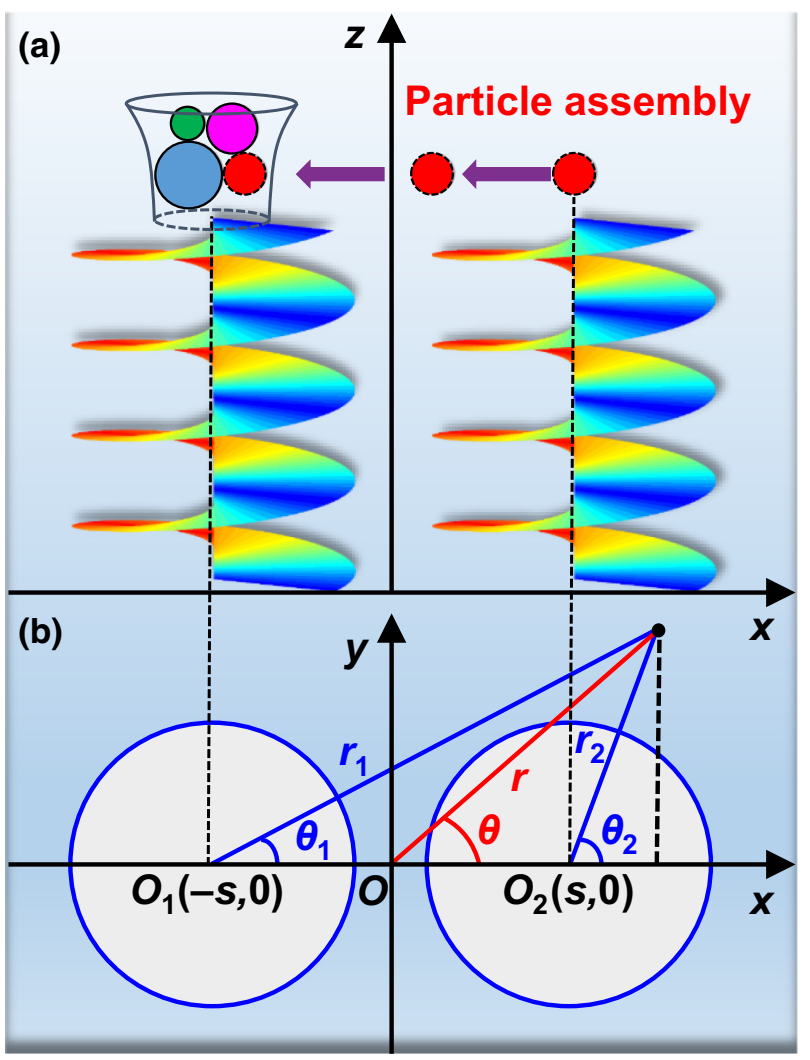

FIG. 1. Schematic of two cylindrical Bessel beams. (a) Particle assembly with two vortices. The particles can have different properties and sizes within the Rayleigh regime. (b) Geometrical relationship of radii and azimuthal angles in local and global coordinate systems. 


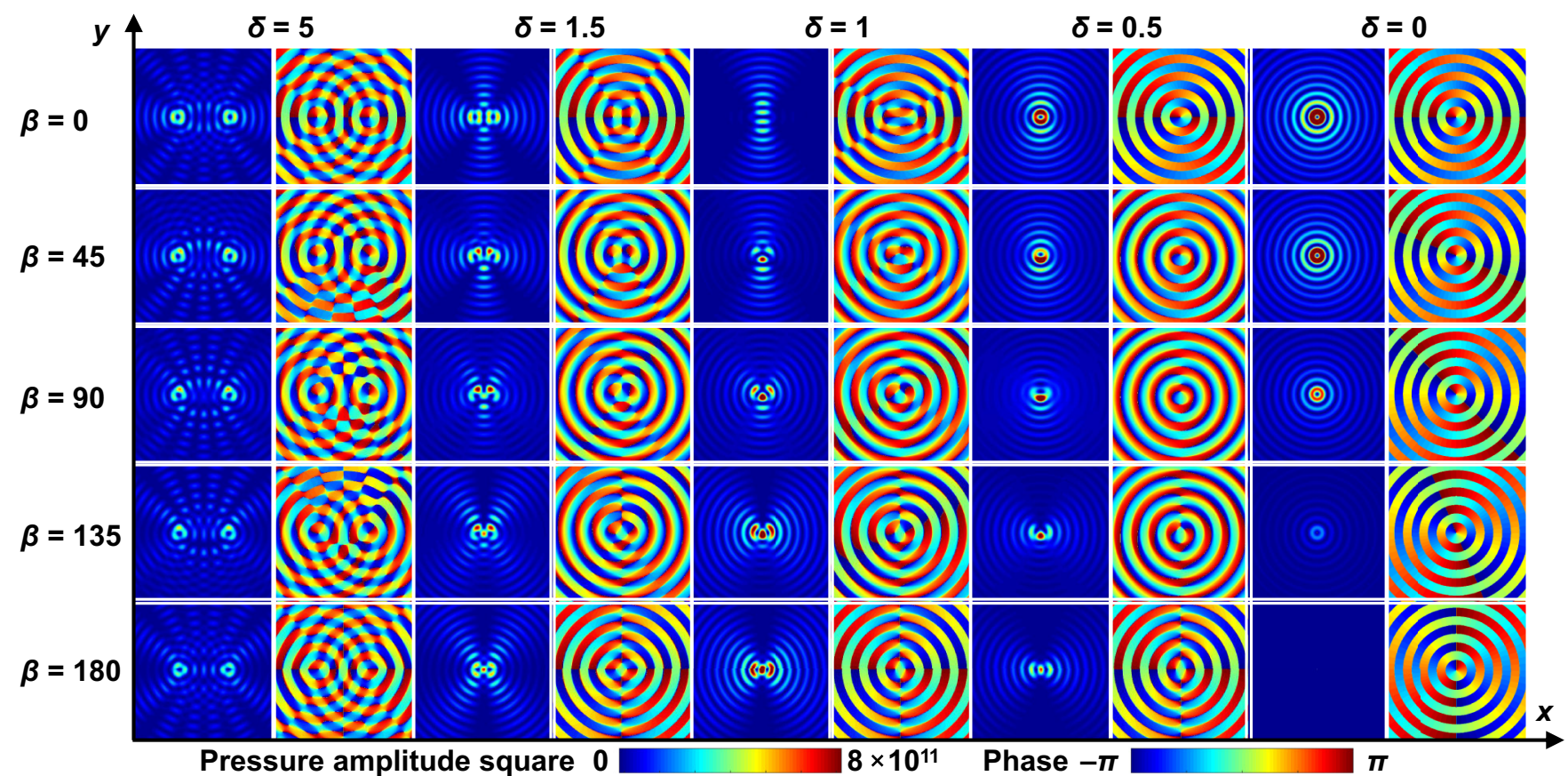

FIG. 2. Square of the pressure amplitude $|p|^{2}$ and phase distribution for two interfering cylindrical Bessel beams with different offset ratios $\delta=s / l_{0}$ (representing the distance between the cores of the two vortices) and phase shifts ( $\beta$ in degrees). The computational domain is $x \in[-4 \lambda, 4 \lambda]$ and $y \in[-4 \lambda, 4 \lambda]$ with $\lambda$ the wavelength. For each $\delta$, the left and right columns are amplitudes and phases of the interfering vortices, respectively. This picture shows that the most optimal case for particle assembly is the in-phase case $(\beta=0)$. Video 1 shows the continuous evolution of the phase and amplitude as a function of $\delta$ in this case (see the Supplemental Material in Ref. [47]).

the acoustic velocity also contributes to the radiation force. This can lead, at small cone angles, to a reverse behavior wherein particles denser and stiffer than the surrounding medium are trapped at the center of Bessel beams of topological order $m=0$, that is to say, at the maximum of the laterally focalized wave [13].

The total pressure field created by the interference of these two vortices is simply the sum $p=p_{1}+p_{2}$. For convenience, we introduce the phase shift between these two vortices $\beta=\beta_{2}-\beta_{1}$ (set $\beta_{1}=0, \beta_{2}=\beta$ in the previous equation) and introduce the local $\left(r_{j}, \theta_{j}\right)$ and global coordinates $(r, \theta)$ linked by the equation

$$
r_{1,2} e^{i \theta_{1,2}}=r e^{i \theta} \pm s
$$

The local coordinates are centered on the central axis $j$ of each vortex and the global coordinates are centered in between the axis of the two vortices [see Fig. 1(b)]. By substituting Eq. (2) into Eq. (1), the total complex pressure in a transverse plane (here $z=0$ for convenience) reads

$$
\begin{aligned}
p= & {\left[A_{1} \frac{J_{1}\left(k r_{1}\right)}{r_{1}}+A_{2} \frac{J_{1}\left(k r_{2}\right)}{r_{2}} e^{i \beta}\right] r e^{i \theta} } \\
& +\left[A_{1} \frac{J_{1}\left(k_{1}\right)}{r_{1}}-A_{2} \frac{J_{1}\left(k r_{2}\right)}{r_{2}} e^{i \beta}\right] s .
\end{aligned}
$$

For convenience, we quantify the beam intensity with the value of $|p|^{2}$ and the phase of the total field is simply the argument of the complex pressure $\arg (p)$. Figure 2 shows the square of the pressure amplitude $|p|^{2}$ and phase distributions of the superposition of two cylindrical Bessel beams as a function of the dimensionless offset ratio $\delta=s / l_{0}$ and the original phase shift $\beta$, with $s$ the distance between the beam axis $O_{1,2}$ and the origin $O$ (see Fig. 1) and $l_{0}$ the distance between the maximum pressure amplitude on the first ring and the origin of a single vortex beam (corresponding to the position of the first maximum of the cylindrical Bessel function). The acoustic frequency is $f=5 \mathrm{MHz}$ and the beam amplitudes are $A_{1,2}=A=10^{6} \mathrm{~Pa}$ throughout the paper unless mentioned otherwise. We can notice that this beam amplitude $A$ leads to a maximum value of the pressure on the first ring $p_{\max }=A \times J_{1}^{\max } \approx 0.6 \times 10^{6} \mathrm{~Pa}$. The computational domain is $x \in[-4 \lambda, 4 \lambda]$ and $y \in[-4 \lambda, 4 \lambda]$, where $\lambda=301 \mu \mathrm{m}$ in water at the frequency considered in this paper with the acoustic parameter listed in Table I.

The results presented in Fig. 2 show that (i) the vortex cores (and hence tweezer traps) are located on the $x$ axis in the in-phase $\left(\beta=0^{\circ}\right)$ and out-of-phase $\left(\beta=180^{\circ}\right)$ cases, while they deviate from the individual core-core line for other phase shifts, such as $\beta=45^{\circ}, 90^{\circ}$, or $135^{\circ}$. Even in the in-phase case, the positions of the composite 
TABLE I. Acoustic parameters for typical elastic materials and various fluids. The properties of polystyrene (PS), Pyrex, a representative biological cell, water, and glycerol are taken from Ref. [48]; the ones for ethanol are from the Engineering ToolBox. The viscosities of blood plasma (BP) and ethanol are from Ref. [49], the density is from Ref. [50], and the sound speed is from Ref. [51].

\begin{tabular}{lcccc}
\hline \hline Material & $\begin{array}{c}\text { Density } \rho_{0} \\
\left(\mathrm{~kg} / \mathrm{m}^{3}\right)\end{array}$ & $\begin{array}{c}\text { Compress. } \kappa \\
(1 / \mathrm{TPa})\end{array}$ & $\begin{array}{c}\text { Longitud. speed of sound } c \\
(\mathrm{~m} / \mathrm{s})\end{array}$ & $\begin{array}{c}\text { Viscosity } \eta \\
(\mathrm{mPa})\end{array}$ \\
\hline PS & 1050 & 172 & 2350 & $\ldots$ \\
Pyrex & 2230 & 27.8 & 5674 & $\ldots$ \\
Cell & 1100 & 400 & 1500 & $\ldots$ \\
Water & 998.2 & 456 & 1482 & 1.002 \\
BP & 1025 & 407 & 1549 & 1.43 \\
Ethanol & 785 & 973 & 1144 & 1.10 \\
Glycerol & 1261 & 219 & 1904 & 1412 \\
\hline \hline
\end{tabular}

vortex cores (resulting from the interference between the two vortices) do not correspond to the positions of the axes of each individual vortex synthesized separately (as we see in Sec. III A). These results are consistent with the work of Maleev and Groover [52], who studied the phase singularities resulting from the interaction of two optical vortices. (ii) When the two vortex axes coincide $(\delta=0)$, the interference pattern evolves entirely from the constructive interference in the in-phase case $\left(\beta=0^{\circ}\right)$, wherein the ring amplitudes sum up, and the completely destructive case when the two vortices are in opposite phases $\left(\beta=180^{\circ}\right)$, leading to a complete canceling of the pressure amplitude. (iii) The in-phase case $\left(\beta=0^{\circ}\right)$ is the most suited configuration for particle assembly since it produces the largest and most isotropic rings surrounding the two vortex cores. (iv) Most importantly, the lateral destructive interference between two in-phase vortex beams when $\delta \rightarrow 1$ creates a path between the repulsive rings that might enable the assembly of two particles (see Video 1 for a continuous description of the evolution of the amplitude and phase as a function of $\delta$ ). Nevertheless, since the situation $\delta \rightarrow 1$ also leads to the weakest pressure gradient in the $x$ direction, further investigation is still necessary to determine whether this gradient is sufficient to maintain a trap along this direction and determine the limited speed at which two particles could be assembled in this configuration.

\section{PARTICLE TRAPPING WITH SYNCHRONIZED VORTICES}

For this purpose, we examine the forces exerted on particles trapped at the core of two synchronized acoustical vortices $\left(\beta=0^{\circ}\right)$ based on Gor'kov's expression [53] of the radiation force in the long-wavelength regime. Indeed, Gor'kov demonstrated that this force can be expressed, for a standing wave, as the gradient of a potential, now referred to as the Gor'kov potential. In this section, we compute this potential and the resulting lateral forces (in the $x$ direction) exerted on two particles initially trapped at the center of two cylindrical acoustical vortices. We then compare this force to the Stokes drag in order to determine the critical velocity $v_{\text {cr }}$ at which two particles can be assembled by moving the two tweezers laterally. Indeed, if the Stokes drag exceeds the radiation force, the particles can be expelled from the trap. Finally, we perform a parametric study of this critical velocity for different typical elastic materials and fluids.

\section{A. Gor'kov potential and force}

For the estimation of the force, we first consider some polystyrene (PS) particles of small radius $a=5 \mu \mathrm{m}$ compared to the wavelength $\lambda=301 \mu \mathrm{m}$, in the range of the validity of Gor'kov theory $(\mathrm{ka} \approx 1 / 10 \ll 1)$, with $k$ the wavenumber. These particles are chosen to demonstrate that particle assembly is possible with this method even for low acoustical contrast with the surrounding fluid (see acoustic parameters listed in Table I). Following Gor'kov [53], the radiation force $\mathbf{F}$ can be expressed as the negative gradient of the potential $U$,

$$
\mathbf{F}=-\nabla U
$$

with

$$
U=2 \pi a^{3} \rho_{0}\left(\frac{\left\langle p^{2}\right\rangle}{3 \rho_{0}^{2} c_{0}^{2}} f_{1}-\frac{\left\langle\mathbf{v}^{2}\right\rangle}{2} f_{2}\right),
$$

where $f_{1}=1-\tilde{\kappa}$ (with the compressibility ratio $\tilde{\kappa}=$ $\left.\kappa_{p} / \kappa_{0}\right)$ and $f_{2}=2(\tilde{\rho}-1) /(2 \tilde{\rho}+1)$ (with density ratio $\left.\tilde{\rho}=\rho_{p} / \rho_{0}\right)$ are the respective contributions of the monopolar and dipolar vibrations of the particle [54]. The compressibility of an elastic particle is $\kappa_{p}=1 / K_{p}$ with $K_{p}=\rho_{p}\left(c_{l}^{2}-4 / 3 c_{t}^{2}\right)$ the bulk modulus, while the compressibility of the fluid is $\kappa_{0}=1 / K_{0}$ with $K_{0}=\rho_{0} c_{0}^{2}$ the bulk modulus of the fluid. In these equations, $\rho_{0}$ and $\rho_{p}$ represent the fluid and particle densities; $c_{0}$ the acoustic velocity in the fluid; $c_{l}$ and $c_{t}$ the longitudinal and transverse velocities of elastic particles, respectively; and finally, $p$ and $\mathbf{v}$ the pressure and velocity associated with the total incident acoustic field in the fluid, 

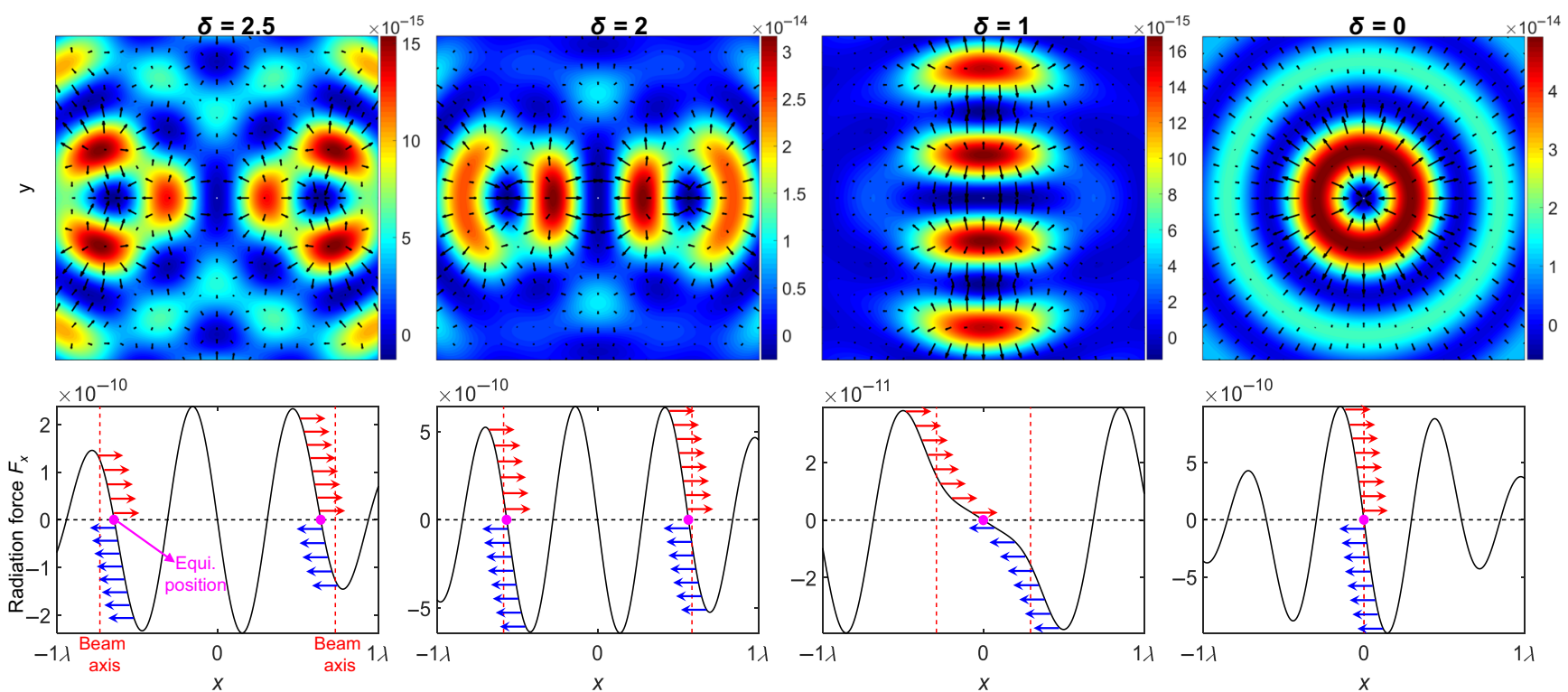

FIG. 3. Upper rows: Gor'kov potential (color) and radiation forces (arrows) resulting from the interaction of two interacting acoustical vortices (of amplitudes $A=10^{6} \mathrm{~Pa}$ and driving frequency $f=5 \mathrm{MHz}$ ) with PS spheres of $5 \mu \mathrm{m}$ for different offset ratios $\delta=s / l_{o}$ (dimensionless distance between the two vortices). Lower row: Lateral forces $F_{x}$ (in newtons) applied to the particles as a function of $x$. The static equilibrium positions (traps) correspond to the null force values in regions of negative force gradient $\left(\nabla F_{x}<0\right)$. The individual vortex cores (in the absence of interference) are represented with a red dashed line. Video 2 shows the continuous evolution of the Gor'kov potential and radiation forces from $\delta=5$ to $\delta=0$ (see the Supplemental Material in Ref. [47]).

respectively. Note that the expression of $f_{1}$ in Gor'kov's original paper [53], based solely on the longitudinal velocity inside the particle, only applies for liquid spheres. The velocity vector is related to the complex pressure, as $\mathbf{v}=-i /\left(\rho_{0} \omega\right) \nabla p$ with $\nabla=\partial / \partial r \mathbf{e}_{r}+\partial / r \partial \theta \mathbf{e}_{\theta}$ the gradient operator in cylindrical coordinates (the term $\partial / \partial z \mathbf{e}_{z}$ is omitted here since the pressure of a nonpropagating cylinder Bessel beam is independent of $z$ ), leading for each vortex to

$$
\begin{aligned}
\mathbf{v}_{j}= & -i \frac{1}{\rho \omega} \nabla p_{j}=-i A_{j} e^{i \beta_{j}} \frac{1}{\rho \omega}\left\{\frac{\partial}{\partial r}\left[J_{1}\left(k r_{j}\right) \frac{r e^{i \theta} \pm s}{r_{j}}\right] \mathbf{e}_{r}\right. \\
& \left.+\frac{\partial}{r \partial \theta}\left[J_{1}\left(k r_{j}\right) \frac{r e^{i \theta} \pm s}{r_{j}}\right] \mathbf{e}_{\theta}\right\}
\end{aligned}
$$

In this expression, the sign before $s$ is + for $j=1$ and - for $j=2$. As previously, if we set $\beta_{1}=0$, we have $\beta_{2}=\beta$. To compute the derivative of the expressions in Eq. (5) with respect to $r$ and $\theta$, we use the trigonometric relations (see Fig. 1): $r_{1}^{2}=r^{2}+s^{2}+2 r s \cos \theta$ and $r_{2}^{2}=$ $r^{2}+s^{2}-2 r s \cos \theta$. Substituting Eqs. (3) and (5) into the expression of the Gor'kov potential in Eq. (4) leads to the final expression of the force given in the Appendix.

The 2D Gor'kov potential and its corresponding negative gradient for two synchronized vortices are depicted in the first row of Fig. 3 for offset ratios $\delta=2.5,2$, 1, and 0 . These figures show that particles can be trapped separately in the two vortices, when the offset is relatively large (in agreeement with the criterion provided in Ref. [13]) and can also be assembled in the center of the two coaxial beams when $\delta=0$. To further demonstrate the feasibility of a structure with two synchronized vortices for particle assembly, the lateral radiation forces in the $x$ direction are calculated and presented in the bottom row of Fig. 3. The particles get trapped when the static equilibrium positions (zero force) are located in the region of the negative force gradient, with the restoring forces pushing them back to the equilibrium positions. This can be observed in Fig. 3, where the red arrows describe positive forces while the blue ones the negative forces, which both push the particles (magenta solid spheres) back to the trapping well. We can note that these static equilibrium positions (marked as magenta solid circles) differ from the position of the individual vortex axes (marked as red dashed lines) when the two vortex beams are not coaxial, due to the interference between the two vortices.

The Gor'kov potential and dynamic motion of the equilibrium positions for offsets ranging from $\delta=5$ to $\delta=0$ are further shown in Video 2 of the Supplemental Material [47], which demonstrates the ability of the synchronized vortices to assemble particles.

\section{B. Radiation and critical Stokes drag forces}

In the previous section, we calculate the quasistatic equilibrium positions of the particles (when the velocities of the tweezer traps tend toward 0 ). In practice, it is, of course, necessary to move particles with a finite velocity, which 


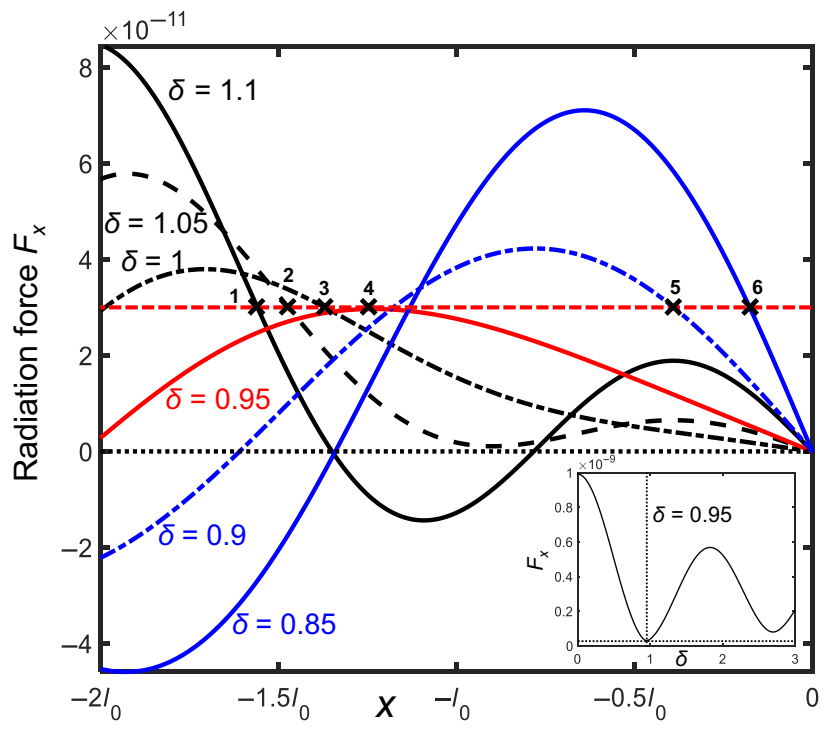

FIG. 4. Lateral radiation force $F_{x}$ (in newtons) applied to PS spheres immersed in water by two interfering vortex beams with offset ratios ranging from $\delta=1.1$ to $\delta=0.85$. Inset: Trapping force as a function of the offset ratio $\delta$. The graph only shows the force for particles located in the half plane $x<0$ on the $x$ axis. Results for particles located in the half plane $x>0$ can be inferred from these data by simple symmetry. The red dashed line describes the critical radiation force at $\delta=0.95$, which is balanced by the Stokes drag force to determine the critical velocity $v_{\mathrm{cr}}$ of the moving tweezers.

leads to the existence of a drag force applied by the fluid on the particles. This drag force can expel the particle from the trap if the radiation force is not sufficient to counteract it. Hence, it is essential to determine the speed limit at which two particles can be assembled. To compute this critical velocity, we assume that (i) the particles are moved by the tweezers at a constant velocity $v_{\text {cr }}$ and (ii) the flow around the particles is in the low Reynolds regime $(\operatorname{Re} \ll 1)$. Considering the small size of the particles $(a=5 \mu \mathrm{m})$, this assumption holds for particles velocities: $v_{\text {cr }} \ll \eta / \rho a=$ $0.2 \mathrm{~m} \mathrm{~s}^{-1}$ in water, with $\eta$ the fluid dynamic viscosity. This value is several orders of magnitude larger than the typical velocities used for microparticle manipulation and, thus, the low Reynolds hypothesis is consistent. Under this circumstance and assuming spherical particles, the magnitude of the drag force is simply the Stokes drag: $F_{d}=6 \pi \eta a v_{\mathrm{cr}}$.

A particle is expelled from the left (right) trap when the drag force exceeds the value of the lateral radiation force $F_{x}$ on the peak located to the left (right) of the static equilibrium position, which we call the "trapping force." When two vortices are approached, Fig. 3 shows that the trapping force becomes minimal when $\delta \approx 1$. This value can be refined numerically by evaluating the trapping force for different offsets $\delta$ and identifying the minimum value (see the inset of Fig. 4). Simulations give a minimum trapping force for a distance between the vortex cores $\delta=0.95$.
Hence, the maximum critical velocity at which two particles can be assembled (assuming that the particles move at a constant speed) is obtained when the drag force is equal to the value of the trapping force $F_{x}^{\text {peak }}(\delta=0.95)$. This leads to the critical velocity:

$$
v_{\mathrm{cr}}=F_{x}^{\text {peak }}(\delta=0.95) / 6 \pi \eta .
$$

To further demonstrate the ability of particle assembly at or near the critical offset, we compute the radiation force along the $x$ axis $F_{x}$ versus the $x$ coordinates in the half plane $x \in\left[-2 l_{0}, 0\right]$, with the offset ratios ranging from $\delta=1.1$ to $\delta=0.85$ with increment $\Delta \delta=0.05$, as shown in Fig. 4 (the values of $F_{x}$ in the other half plane can be obtained by symmetry). The successive dynamic equilibrium positions of one of the trapped particles at the critical speed $v_{\text {cr }}$ (numbered from 1 to 6) are represented by black crosses, when the offset is reduced from $\delta=1.1$ to $\delta=0.85$.

As observed, the particle can be successively moved and then brought to a single centered trap, when the two vortex beams approach each other.

\section{Critical velocity: Parametric study}

In Sec. III B, we determine the expression of the critical velocity for particle assembly with two synchronized vortices for PS spheres. We now extend these results to different particles, fluids, and input powers, with the acoustic parameters listed in Table I. In particular, we determine from Eq. (6) the critical velocity for the following particles: PS, Pyrex, a representative biological cell, and surrounding fluids (water, ethanol, BP, and glycerol). The evolution of the critical velocity $v_{\mathrm{cr}}$ as a function of the input power $A^{2}$ for different particles in water is represented in the inset of Fig. 5(a). The beam amplitude varies from $A=0 \mathrm{MPa}$ to $A=2 \mathrm{MPa}$. Obviously, the critical velocity is proportional to $A^{2}$. Indeed, the Gor'kov potential in Eq. (4) depends on the terms $\left\langle p^{2}\right\rangle$ and $\left\langle\mathbf{v}^{2}\right\rangle$, which are both proportional to the square of the beam amplitude $A$ [see Eqs. (3) and (5)]. Then since the critical velocity can be expressed as $v_{\mathrm{cr}}=-\partial U / \partial x(\delta=0.95) /(6 \pi \eta a)$, it is also proportional to $A^{2}$. The values of the critical velocities for the assembly of small PS and Pyrex particles and typical cells in water with a driving pressure amplitude $A$ of $2 \mathrm{MPa}$ are compatible with experiments in microchannels. To broaden this analysis to any type of particle, we plot a 2D graph [Fig. 5(a)], featuring the values of the critical velocity in water as a function of the particle compressibility and density at the fixed frequency $f=5 \mathrm{MHz}$ and amplitude $A=1 \mathrm{MPa}$. These results show, as expected, that cells are one of the most difficult objects to address, owing to their low acoustic contrast. Nevertheless, they can still be moved and assembled with reasonable speeds of several hundreds microns per second at acoustic levels that remain harmless for living objects (far below the 

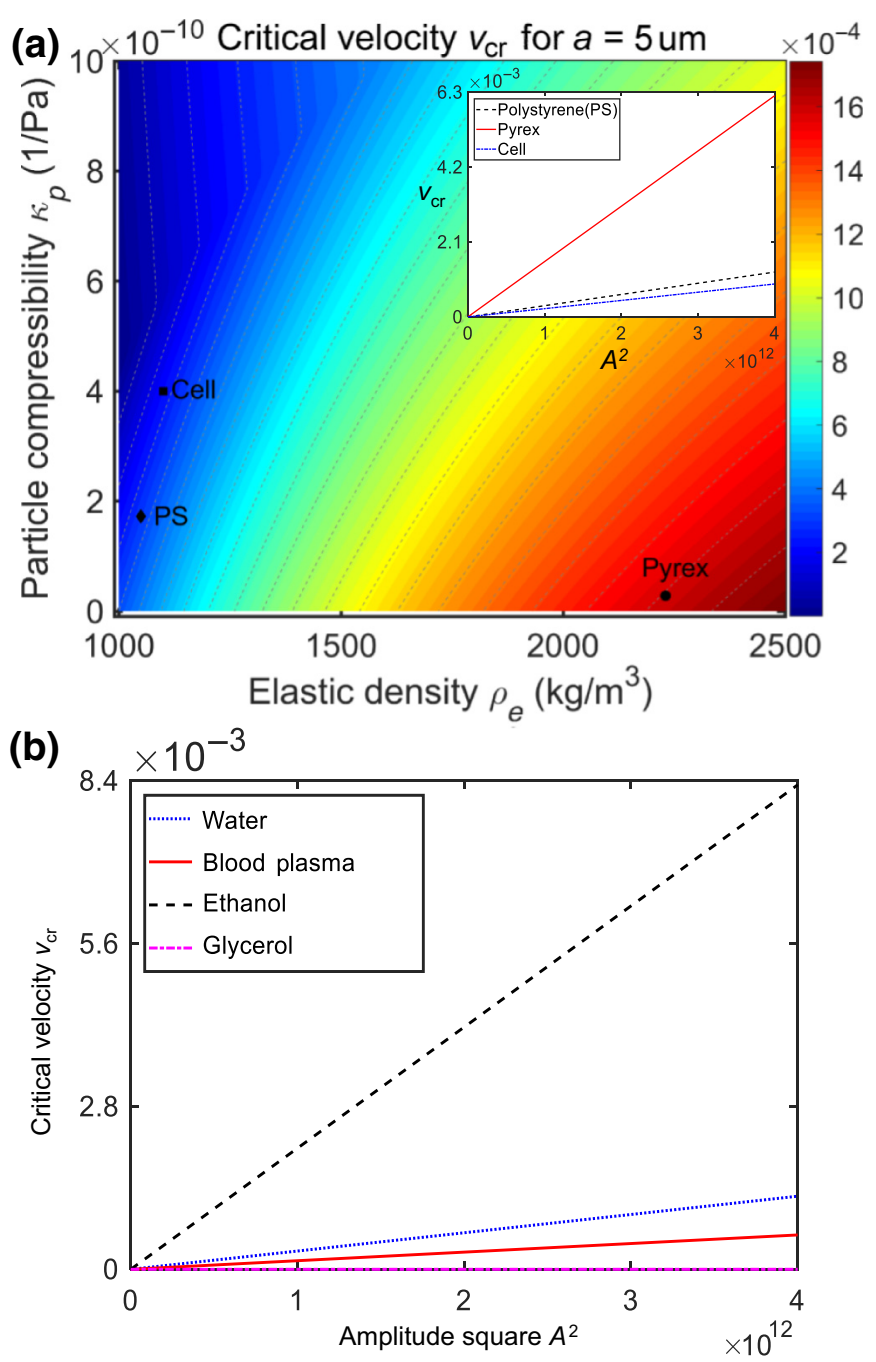

FIG. 5. (a) Critical velocity for the assembly of a 5- $\mu$ m elastic sphere embedded in water as a function of the particle density and compressibility at a fixed pressure amplitude $A=10^{6} \mathrm{~Pa}$ and driving frequency $f=5 \mathrm{MHz}$. Inset: Critical velocity for four specific materials as a function of the square of the acoustic wave pressure amplitude $A^{2}$. (b) Critical velocity for a 5- $\mu \mathrm{m}$ PS sphere embedded in different liquids as a function of the square of the acoustic wave pressure amplitude $A^{2}$.

cavitation threshold and power levels used, e.g., for echography). Finally, we compute the critical velocity for PS particles embedded in different common fluids [Fig. 5(b)] as a function of the driving pressure. This time, three properties of the fluid influence the critical velocity: the acoustic wave speed, the fluid density influencing the radiation force, and the viscosity of the fluid influencing the Stokes drag.

\section{DISCUSSION AND CONCLUSION}

In this paper, we demonstrate theoretically that two synchronized cylindrical vortex beams are suitable to move and assemble particles with sizes much smaller than the wavelength in 2D. (Note that the radiation forces of particles with arbitrary sizes could be obtained by adding the beam shape coefficients of incidence and combining with the radiation stress tensor method [18,31]). Indeed, the interference between two neighboring vortex beams creates an attractive path between the two initial rings used for particle trapping. This enables us to merge the particles without the need to cross the potential barrier of the ring. The maximum critical velocities for particle assembly are determined based on the balance between the radiation and drag forces. Indeed, the former maintains the particle in the trap, while the latter tends to push it out of the trap. The estimated speeds are compatible with the manipulation of microparticles in microchannels, even for particles with low contrast such as cells, at acoustic levels compatible with the manipulation of living objects. This work constitutes a step toward particle assembly with acoustical tweezers, which is different from the assembling method using the short-range attraction between particles $[55,56]$. Potential continuation of this work includes the extension to 3D particle assembly, in particular beyond the long-wavelength regime and the consideration of particle rotation.

\section{ACKNOWLEDGMENTS}

We acknowledge the support of the ERC Generator and Prematuration programs funded by ISITE Université Lille Nord-Europe.

\section{APPENDIX: EXPRESSIONS OF VELOCITY AND RADIATION FORCE}

The velocity expressions of the individual vortex (set $\beta_{1}=0, \beta_{2}=\beta$ throughout the paper) are

$$
\mathbf{v}_{1}=-i \frac{1}{\rho \omega} A_{1}\left\{C_{1} \mathbf{e}_{r}+B_{1} \mathbf{e}_{\theta}\right\}
$$

and

$$
\mathbf{v}_{2}=-i \frac{1}{\rho \omega} A_{2} e^{i \beta}\left\{C_{2} \mathbf{e}_{r}+B_{2} \mathbf{e}_{\theta}\right\},
$$

with the coefficients $C_{1}, B_{1}, C_{2}$, and $B_{2}$ :

$$
\begin{aligned}
C_{1}= & \frac{\left[J_{1}^{\prime}\left(k r_{1}\right) k r_{1}-J_{1}\left(k r_{1}\right)\right](r+s \cos \theta)}{r_{1}^{3}}\left(r e^{i \theta}+s\right) \\
& +\frac{J_{1}\left(k r_{1}\right)}{r_{1}} e^{i \theta}, \\
B_{1}= & \frac{-\left[J_{1}^{\prime}\left(k r_{1}\right) k r_{1}-J_{1}\left(k r_{1}\right)\right] s r \sin \theta}{r_{1}^{3}}\left(e^{i \theta}+\frac{s}{r}\right) \\
& +\frac{J_{1}\left(k r_{1}\right)}{r_{1}} i e^{i \theta},
\end{aligned}
$$




$$
\begin{aligned}
C_{2}= & \frac{\left[J_{1}^{\prime}\left(k r_{2}\right) k r_{2}-J_{1}\left(k r_{2}\right)\right](r-s \cos \theta)}{r_{2}^{3}}\left(r e^{i \theta}-s\right) \\
& +\frac{J_{1}\left(k r_{2}\right)}{r_{2}} e^{i \theta}, \\
B_{2}= & \frac{\left[J_{1}^{\prime}\left(k r_{2}\right) k r_{2}-J_{1}\left(k r_{2}\right)\right] s r \sin \theta}{r_{2}^{3}}\left(e^{i \theta}-\frac{s}{r}\right) \\
& +\frac{J_{1}\left(k r_{2}\right)}{r_{2}} i e^{i \theta} .
\end{aligned}
$$

The total velocity vector is $\mathbf{v}=\mathbf{v}_{1}+\mathbf{v}_{2}=v_{r} \mathbf{e}_{r}+v_{\theta} \mathbf{e}_{\theta}$, with the time average

$$
\left\langle\mathbf{v}^{2}\right\rangle=\frac{1}{2} \operatorname{Re}\left\{v_{r} v_{r}^{*}+v_{\theta} v_{\theta}^{*}\right\}
$$

where $v_{r}=-i(1 / \rho \omega) \times\left(A_{1} C_{1}+A_{2} e^{i \beta} C_{2}\right)$ and $v_{\theta}=$ $-i(1 / \rho \omega) \times\left(A_{1} B_{1}+A_{2} e^{i \beta} B_{2}\right)$. Note that there is an axial velocity component $v_{z}$ for propagating Bessel beams when the cone angle $\gamma$ is not $90^{\circ}$. The time average of pressure squared could be expressed as $\left\langle p^{2}\right\rangle=\frac{1}{2} \operatorname{Re}\left\{p p^{*}\right\}$. The force can be simply obtained by substituting the above equations into Gorkov's potential in Eq. (4).

[1] B. T. Hefner and P. L. Marston, An acoustical helicoidal wave transducer with applications for the alignment of ultrasonic and underwater systems, J. Acoust. Soc. Am. 106, 3313 (1999).

[2] P. L. Marston, Scattering of a bessel beam by a sphere: II. Helicoidal case and spherical shell example, J. Acoust. Soc. Am. 124, 2905 (2008).

[3] D. Baresh, J. L. Thomas, and R. Marchiano, Spherical vortex beams of high radial degree for enhanced single-beam tweezers, J. Appl. Phys. 113, 184901 (2013).

[4] C. R. P. Courtney, C. E. M. Demore, H. Wu, A. Grinenko, P. D. Wilcox, S. Cochran, and B. W. Drinkwater, Independent trapping and manipulation of microparticles using dexterous acoustic tweezers, Appl. Phys. Lett. 104, 154103 (2014).

[5] A. Marzo, S. A. Seah, B. W. Drinkwater, D. R. Sahoo, B. Long, and S. Subramanian, Holographic acoustic elements for manipulation of levitated objects, Nat. Commun. 6, 8661 (2015).

[6] J. L. Thomas, R. Marchiano, and D. Baresch, Acoustical and optical radiation pressure and the development of single beam acoustical tweezers, J. Quant. Spectrosc. Ra. 195, 55 (2017).

[7] A. Riaud, M. Baudoin, O. B. Matar, L. Becerra, and J. L. Thomas, Selective Manipulation of Microscopic Particles with Precursor Swirling Rayleigh Waves, Phys. Rev. Appl. 7, 024007 (2017).

[8] A. Marzo and B. W. Drinkwater, Holographic acoustic tweezers, P. Natl. Acad. Sci. U.S.A. 116, 84 (2019).
[9] M. Baudoin, J. C. Gerbedoen, M. O. Bou, N. Smagin, A. Riaud, and J. L. Thomas, Folding a focalized acoustical vortex on a flat holographic transducer: Miniaturized selective acoustical tweezers, Sci. Adv. 5, eaav1967 (2019).

[10] J. Durnin, Exact solutions for nondiffracting beams. I. The scalar theory, J. Opt. Soc. Am. A 4, 651 (1987).

[11] J. Durnin, J. J. Miceli, Jr., and J. H. Eberly, Diffraction-free Beams, Phys. Rev. Lett. 58, 1499 (1987).

[12] P. L. Marston, Scattering of a bessel beam by a sphere, J. Acoust. Soc. Am. 121, 753 (2007).

[13] X. D. Fan and L. Zhang, Trapping Force of Acoustical Bessel Beams on a Sphere and Stable Tractor Beams, Phys. Rev. Appl. 11, 014055 (2019).

[14] G. T. Silva, J. H. Lopes, and F. G. Mitri, Off-axial acoustic radiation force of repulsor and tractor bessel beams on a sphere, IEEE Trans. Ultrason. Ferroelectr. Freq. Control 60, 1207 (2013).

[15] P. L. Marston, Negative axial radiation forces on solid spheres and shells in a bessel beam, J. Acoust. Soc. Am. 122, 3162 (2007).

[16] P. L. Marston, Radiation force of a helicoidal bessel beam on a sphere, J. Acoust. Soc. Am. 125, 3539 (2009).

[17] L. Zhang and P. L. Marston, Geometrical interpretation of negative radiation forces of acoustical bessel beams on spheres, Phys. Rev. E 84, 035601 (2011).

[18] Z. Gong, P. L. Marston, W. Li, and Y. Chai, Multipole expansion of acoustical bessel beams with arbitrary order and location, J. Acoust. Soc. Am. 141, EL574 (2017).

[19] L. Zhang, A general theory of arbitrary bessel beam scattering and interactions with a sphere, J. Acoust. Soc. Am. 143, 2796 (2018).

[20] D. Baresch, J. L. Thomas, and R. Marchiano, Observation of a Single-beam Gradient Force Acoustical Trap for Elastic Particles: Acoustical Tweezers, Phys. Rev. Lett. 116, 024301 (2016).

[21] A. Riaud, J. L. Thomas, M. Baudoin, and M. O. Bou, Taming the degeneration of bessel beams at anisotropicisotropic interface: Toward three-dimensional control of confined vortical waves, Phys. Rev. E 92, 063201 (2015).

[22] L. Zhang and P. L. Marston, Angular momentum flux of nonparaxial acoustic vortex beams and torques on axisymmetric objects, Phys. Rev. E 84, 065601 (2011).

[23] G. T. Silva, T. P. Lobo, and F. G. Mitri, Radiation torque produced by an arbitrary acoustic wave, EPL 97, 54003 (2012).

[24] L. Zhang and P. L. Marston, Acoustic radiation torque on small objects in viscous fluids and connection with viscous dissipation, J. Acoust. Soc. Am. 136, 2917 (2014).

[25] L. Zhang, Reversals of Orbital Angular Momentum Transfer and Radiation Torque, Phys. Rev. Appl. 10, 034039 (2018).

[26] Z. Gong, P. L. Marston, and W. Li, Reversals of Acoustic Radiation Torque in Bessel Beams Using Theoretical and Numerical Implementations in Three Dimensions, Phys. Rev. Appl. 11, 064022 (2019).

[27] D. Baresch, Acoustical tweezers, Ph.D. thesis, Université Pierre et Marie Curie, 2014.

[28] A. Marzo, M. Caleap, and B. W. Drinkwater, Acoustic Virtual Vortices with Tunable Orbital Angular Momentum for 
Trapping of mie Particles, Phys. Rev. Lett. 120, 044301 (2018).

[29] D. Baresch, J. L. Thomas, and R. Marchiano, Orbital Angular Momentum Transfer to Stably Trapped Elastic Particles in Acoustical Vortex Beams, Phys. Rev. Lett. 121, 074301 (2018).

[30] Z. Gong, Study on acoustic scattering characteristics of objects in Bessel beams and the related radiation force and torque, Ph.D. thesis, Huazhong University of Science \& Technology, 2018.

[31] Z. Gong, P. L. Marston, and W. Li, T-matrix evaluation of three-dimensional acoustic radiation forces on nonspherical objects in bessel beams with arbitrary order and location, Phys. Rev. E 99, 063004 (2019).

[32] J. L. Thomas and R. Marchiano, Pseudo Angular Momentum and Topological Charge Conservation for Nonlinear Acoustical Vortices, Phys. Rev. Lett. 91, 244302 (2003).

[33] R. Marchiano and J. L. Thomas, Doing Arithmetic with Nonlinear Acoustic Vortices, Phys. Rev. Lett. 101, 064301 (2008).

[34] A. Riaud, J. L. Thomas, E. Charron, A. Bussonnière, O. B. Matar, and M. Baudoin, Anisotropic Swirling Surface Acoustic Waves from Inverse Filtering for On-chip Generation of Acoustic Vortices, Phys. Rev. Appl. 4, 034004 (2015).

[35] M. D. Guild, C. J. Naify, T. P. Martin, C. A. Rohde, and G. J. Orris, Superresolution through the topological shaping of sound with an acoustic vortex wave antenna, arXiv:1608.01887, 2016.

[36] A. Riaud, M. Baudoin, J. L. Thomas, and O. B. Matar, Saw synthesis with idts array and the inverse filter: Toward a versatile saw toolbox for microfluidics and biological applications, IEEE Trans. Ultrason. Ferroelectr. Freq. Control 63, 1601 (2016).

[37] N. Jiménez, R. Picó, V. Sánchez-Morcillo, V. RomeroGarcía, L. M. García-Raffi, and K. Staliunas, Formation of high-order acoustic bessel beams by spiral diffraction gratings, Phys. Rev. E 94, 053004 (2016).

[38] T. Wang, M. Ke, W. Li, Q. Yang, C. Qiu, and Z. Liu, Particle manipulation with acoustic vortex beam induced by a brass plate with spiral shape structure, Appl. Phys. Lett. 109, 123506 (2016).

[39] N. Jimenez, V. Romero-Garcia, L. M. Garzcia-Raffi, F. Camarena, and K. Staliunas, Sharp acoustic vortex focusing by fresnel-spiral zone plates, Appl. Phys. Lett. 112, 204101 (2018).

[40] X. Jiang, Y. Li, B. Liang, J. C. Cheng, and L. Zhang, Convert Acoustic Resonances to Orbital Angular Momentum, Phys. Rev. Lett. 117, 034301 (2016).
[41] L. Ye, C. Qiu, J. Lu, K. Tang, H. Jia, M. Ke, S. Peng, and Z. Liu, Making sound vortices by metasurfaces, Aip Adv. 6, 085007 (2016).

[42] K. Tang, Y. Hong, C. Qiu, S. Peng, M. Ke, and Z. Liu, Making acoustic half-bessel beams with metasurfaces, Jpn. J. Appl. Phys. 55, 110302 (2016).

[43] R. D. Muelas-Hurtado, J. L. Ealo, J. F. Pazos-Ospina, and K. Volke-Sepúlveda, Generation of multiple vortex beam by means of active diffraction gratings, Appl. Phys. Lett. 112, 084101 (2018).

[44] A. Lenshof and T. Laurell, Continuous separation of cells and particles in microfluidic systems, Chem. Soc. Rev. 39, 1203 (2010).

[45] X. Ding, P. Li, Z. S. Stratton, S.-C. Lin, N. Nama, F. Guo, D. Slotcavage, J. Mao, X. Shi, T. J. Huang, and F. Costanzo, Surface acoustic wave microfluidics, Lab Chip 13, 3626 (2013).

[46] A. Ozcelik, J. Rufo, F. Guo, Y. Gu, P. Li, J. Lata, and T. J. Huang, Acoustic tweezers for the life sciences, Nat. Methods 15, 1021 (2018).

[47] See Supplemental Material at http://link.aps.org/supple mental/10.1103/PhysRevApplied.12.024045 for Video 1 and Video 2.

[48] M. Settnes and H. Bruus, Forces acting on a small particle in an acoustical field in a viscous fluid, Phys. Rev. E 85, 016327 (2012).

[49] A. A. M. Bui, A. V. Kashchuk, M. A. Balanant, T. A. Nieminen, H. Rubinsztein-Dunlop, and A. B. Stilgoe, Calibration of force detection for arbitrarily shaped particles in optical tweezers, Sci. Rep. 8, 10798 (2018).

[50] https://hypertextbook.com/facts/2004/MichaelShmukler. shtml.

[51] https://itis.swiss/virtual-population/tissue-properties/datab ase/acoustic-properties/speed-of-sound/.

[52] I. D. Maleev and G. A. Swartzlander, Composite optical vortices, J. Opt. Soc. Am. B 20, 1169 (2003).

[53] L. P. Gor'kov, On the forces acting on a small particle in an acoustical field in an ideal fluid, Sov. Phys. Dokl. 6, 773 (1962).

[54] H. Bruus, Acoustofluidics 7: The acoustic radiation force on small particles, Lab Chip 12, 1014 (2012).

[55] M. Wang, C. Qiu, S. Zhang, R. Han, M. Ke, and Z. Liu, Sound-mediated stable configurations for polystyrene particles, Phys. Rev. E 96, 052604 (2017).

[56] M. X. Lim, A. Souslov, V. Vitelli, and H. M. Jaeger, Cluster formation by acoustic forces and active fluctuations in levitated granular matter, Nat. Phys. 15, 460 (2019). 